\title{
Calculation of Thermodynamic Functions for Polyatomic Molecules
}

\author{
Harold W. Woolley
}

\begin{abstract}
Formulas are given from which the thermodynamic functions may be obtained for polyatomic molecules not exhibiting special phenomena, such as internal rotation. The effects of first and second order anharmonicities and rotational-vibrational interaction are treated in detail, as also are the angular momentum effects of doubly degenerate vibrations in linear molecules. This paper presents formulas for the corrections carried to higher order than has hitherto been available.
\end{abstract}

\section{Introduction}

There is occasionally need for accurate thermodynamic functions for polyatomic molecules. A definite calculating procedure exists for approximate calculations via the rigid-rotator harmonic oscillator treatment, with small corrections for effects of anharmonicity, stretching, etc.

This paper presents formulas for the corrections carried to higher order than has hitherto been available. Calculations can thus be made via the approximation formulas with accuracy probably comparable with that of the direct summation method using extrapolated formulas for the energy levels, aside from effects related to vibrational and rotational cut-off, which have been omitted.

\section{Nonlinear Polyatomic Molecules}

The partition function for the internal molecular energy states may be written as

For nonlinear molecules,

$$
Q=Q_{R} Q_{R c} Q_{H} . O . Q_{c} .
$$

$$
Q_{R}=(1 / \gamma) \sqrt{\left(\pi / A_{0} B_{0} C_{0}\right)(k T / h c)^{3}}
$$

is the rigid rotator partition function for the lowest vibrational energy state, where $A_{0}, B_{0}$, and $C_{0}$ are the rotational constants for the ground vibrational state, and $\gamma$ is the symmetry number. $Q_{H . O}$. is the partition function for vibrational levels of harmonic oscillators. $Q_{R c}$ is a correction factor including (1) a factor $1+\rho T$. . . for rotational stretching, as shown by Wilson [1] ${ }^{2}$ and (2) a factor $1+\theta_{1} / T+\theta_{2} / T^{2}$ for a low temperature quantum correction for rotation as shown by Stripp and Kirkwood [2]. ${ }^{3} \quad Q_{c}$ is a factor including rotational-vibrational interaction and the anharmonicity corrections, now to be treated in detail.

The rotational-vibrational interaction in its simple form arises from the vibrational dependence of the analogue of eq (2) for higher vibrational states. Before summation over vibrational quantum numbers, the dependence is introduced by including the factor

$$
R_{v}=\exp \left[\Sigma a_{i} v_{i}+\Sigma a_{i i} v_{i}^{2}+\Sigma a_{i j} v_{i} v_{j}+\Sigma a_{i i i} v_{i}^{3}+\Sigma a_{i i j} v_{i}^{2} v_{j}+\Sigma a_{i j k} v_{i} v_{j} v_{k}\right]
$$

equivalent to a form introduced by Benedict [3]. ${ }^{4}$ With his formulation, the constants in the factor $R_{v}$ come from sums of corresponding constants for $A_{v}, B_{v}$, and $C_{v}$. A summation procedure adequate to treat this with the anharmonicity effects follows, extending the latter beyond the linear terms of Stockmayer, Kavanagh, and Mickley [4].

1 Related to a thesis submitted to the Graduate School of the University of Michigan in partial fulfillment of the requirements for the Ph. D. degree in Physics.

2 Figures in brackets indicate the literature references at the end of this paper.

${ }^{3}$ Expressions representing the $T^{2}$ rotational stretching effect and the $T^{-3}$ low temperature correction as well as a low temperature correction for symmetry effects for different classes of rotational levels for the asymmetric rotator are included in the thesis.

4 An equivalent representation in slightly different form is given in the thesis. 
For a given vibrational level, specified by vibrational quantum numbers $v_{i}, 1 \leq i \leq n$, the statistical weight is the product of the $n$ quantities

$$
p_{i}=\left(v_{i}+d_{i}-1\right) ! /\left[v_{i} !\left(d_{i}-1\right) !\right]
$$

where $d_{i}$ is the degeneracy associated with the $i$ th fundamental frequency.

The summation over the vibrational levels may be indicated as

$$
Q=Q_{R} Q_{R c} \Sigma R_{v_{i}^{\prime} \mathrm{s}} R_{v} \exp \left[-h c G_{v} / k T\right] \prod_{i} p_{i}
$$

with the vibrational energy in wave numbers given by

$$
G_{v}=\Sigma \omega_{i} v_{i}+\Sigma x_{i i} v_{i}\left(v_{i}-1\right)+\Sigma x_{i j} v_{i} v_{j}+\Sigma y_{i i i} v_{i}\left(v_{i}-1\right)\left(v_{i}-2\right)+\Sigma y_{i i j} v_{i}\left(v_{i}-1\right) v_{j}+\Sigma y_{i j k} v_{i} v_{j} v_{k} .
$$

The relations between these constants and the more usual ones are indicated in appendix 1 .

The Boltzmann factor is separated into two factors, one for harmonic oscillators fitting the lowest levels and a second giving the alteration due to anharmonicity

$$
\exp \left[-h c G_{v} / k T\right]=\exp \left[-h c \Sigma \omega_{i} v_{i} / k T\right] \exp \left[-h c\left(G_{v}-\Sigma \omega_{i} v_{i}\right) / k T\right] .
$$

$Q_{v c}$, the product of the second factor on the right hand side of (7) with $R_{v}$, is expanded into a power series whose terms are polynomials in the $v$ 's. Defining a function

it can be shown that ${ }^{5}$

$$
f_{i}(Z)=v_{i} ! /\left(v_{i}-Z\right) !
$$

$$
\sum_{v_{i}=0}^{\infty} p_{i} e^{-u_{i} v_{i}}=\left(1-e^{-u_{i}}\right)^{-d_{i}}
$$

where $u_{i}=h c \omega_{i} / k T$, and that

$$
\sum_{v_{i}=0}^{\infty} f_{i}(Z) p_{i} e^{-u_{i} v_{i}}=\left[\left(d_{i}+Z-1\right) ! /\left(d_{i}-1\right) !\right] \cdot\left[e^{-Z u_{i}} /\left(1-\mathrm{e}^{-u_{i}}\right)^{d_{i}+Z}\right]
$$

The factor $Q_{v c}$ can be expressed as a sum of terms, each linear in whatever $f_{i}$ 's it contains. ${ }^{6}$ The results of multiple summation over all $v_{i}$ 's follow by application of eq $(10)$. If the righthand side of eq (10) be designated by $f_{i}^{*}(Z)$, one has $\Sigma_{v} Q_{v} Q_{v c}=Q_{H . o .} Q_{c}$, where $Q_{v}=\exp \left[-\Sigma_{i} u_{i} v_{i}\right]$ $Q_{H . o .}=\Sigma_{v} Q_{v}$ and where $Q_{c}$ is the same function of $f_{i}^{*}(Z)$ as $Q_{v c}$ was of $f_{i}(Z)$. Thus eq (1) has been evaluated, $Q_{H . O}$. being given by

$$
Q_{H . O .}=\prod_{i}\left(1-e^{-u_{i}}\right)^{-d_{i}} .
$$

There is some advantage in using the logarithm of the partition function from this point on because of analytic cancellations of some higher order terms. The terms removed are cross products without linkage in the subscripts, such as $x_{i j} x_{k l}, x_{i i} x_{j k}$, and $a_{i} x_{j j}{ }^{2}$, for example.

The contribution to In $Q_{c}$ due to $x_{i i}$ and $x_{i j}$ terms, with $r_{i}=e^{-u_{i}}$ and $s_{i}=\left(1-e^{-u_{i}}\right)^{-1}$ is

$$
\begin{aligned}
-(h c / k T) & \sum\left\{x_{i i} d_{i}\left(d_{i}+1\right) r_{i}^{2} s_{i}^{2}+x_{i j} d_{i} d_{j} r_{i} r_{j} s_{i} s_{j}\right\}+\frac{1}{2}(h c / k T)^{2} \sum\left\{2 x_{i i}^{2} d_{i}\left(d_{i}+1\right) r_{i}^{2}\left[1+2\left(d_{i}+1\right) r_{i}\right] s_{i}^{4}\right. \\
& \left.+x_{i j}^{2} d_{i} d_{j} r_{i} r_{j}\left[1+d_{i} r_{i}+d_{j} r_{j}\right] s_{i}^{2} s_{j}^{2}+2 x_{i j} x_{i k} d_{i} d_{j} d_{k} r_{i} r_{j} r_{k} s_{i}^{2} s_{j} s_{k}+4 x_{i i} x_{i j} d_{i}\left(d_{i}+1\right) d_{j} r_{i}^{2} r_{j} s_{i}^{3} s_{j}\right],
\end{aligned}
$$

with terms of higher order for this and other contributions listed in appendix 4. At high temperature the first term varies as $T$ and the second as $T^{2}$. The terms in the appendix are higher than $T^{2}$ in principal dependence at high temperature.

${ }^{5}$ See appendix 2.

${ }^{6}$ Relations giving the simpler polynomials in terms of the $f$ 's will be found in appendix 3. 
Similarly, the contribution of higher order anharmonic terms is

$$
-(h c / k T) \sum\left\{y_{i i i} d_{i}\left(d_{i}+1\right)\left(d_{i}+2\right) r_{i}^{3} s_{i}^{3}+y_{i i j} d_{i}\left(d_{i}+1\right) d_{j} r_{i}^{2} r_{j} s_{i}^{2} s_{j}+y_{i j k} d_{i} d_{j} d_{k} r_{i} r_{j} r_{k} s_{i} s_{j} s_{k}\right\} .
$$

The contribution due to rotational-vibrational interaction is

$$
\sum\left\{a_{i} d_{i} r_{i} s_{i}+a_{i i} d_{i} r_{i}\left(1+d_{i} r_{i}\right) s_{i}^{2}+a_{i j} d_{i} d_{j} r_{i} r_{j} s_{i} s_{j}+\frac{1}{2} a_{i}^{2} d_{i} r_{i} s_{i}^{2}\right\}
$$

as far as $a$ 's alone are concerned, while their mixing with anharmonicities gives

$$
-(h c / k T) \sum\left\{2 a_{i} x_{i i} d_{i}\left(d_{i}+1\right) r_{i}^{2} s_{i}^{3}+a_{i} x_{i j} d_{i} d_{j} r_{i} r_{j} s_{i}^{2} s_{j}\right\} .
$$

In all of these (multiple) summations, if the terms are completely symmetric in the indices that are present, such as $i, j$, etc., the symmetric indices are to progress in size as with $i<j<k$, and so forth. For terms not completely symmetric in the indices, the indices not symmetric are simply not to be equal, thus $i \neq j \neq k$, and so forth. ${ }^{7}$

\section{Linear Molecules}

There is an additional kind of vibrational-rotational interaction, concerning vibrational angular momentum, which is important for linear molecules. When doubly degenerate vibrations occur, the vibrational energy, eq (6), contains an additional term $g=\sum_{i \leq j} g_{i j} l_{i} l_{j}$. For linear molecules, in addition, there is dependence of the rotational energy upon $l$ where $l=\Sigma l_{i}$, giving for the two together

$$
F(J, l)=g+B_{v}\left[J(J+1)-l^{2}\right]-D_{v}\left[J(J+1)-l^{2}\right]^{2},
$$

where $J=l, l+1, l+2$. . ., with double degeneracy for $|l|>0$.

The sum over the rotational levels for $J \geq|l|$ follows from a half integer summation formula of the Euler-Maclaurin type [5]

$\sum_{n=n_{1}}^{\infty} f(n)=\int_{n_{1}-\frac{1}{2}}^{\infty} f(n) d n+(1 / 24) f^{\prime}\left(n_{1}-\frac{1}{2}\right)-(7 / 5760) f^{\prime \prime \prime}\left(n_{1}-\frac{1}{2}\right)+(31 / 967680) f^{(5)}\left(n_{1}-\frac{1}{2}\right) \ldots$.

giving

$$
\sum_{J=|l|}^{\infty}(2 J+1) \exp h c B\left[J(J+1)-l^{2}\right] / k T=k T / h c B+1 / 3+\ldots
$$

and

$$
Q=\sum_{J=|l|}^{\infty}(2 J+1) e^{-h c F / k T}=(1 / \gamma)\left[k T / h c B+1 / 3+2(k T / h \mathrm{c} B)^{2} D / B\right] \cdot e^{-h c g / k T} .
$$

The exponential in $g$ is expanded as a power series and the summations made with $v_{i}$ increasing by steps of two from $l_{i}$ to $\infty$, then summing $l_{i}$ from $-\infty$ to $+\infty$. If $g$ is extended to include possible contributions to $\ln Q_{c}$ varying as $T^{2}$ at high temperature, while retaining the $g_{i j}$ term needed for nonlinear molecules, as in

$$
g=\sum_{i} g_{i i} l_{i}^{2}+\sum_{i<j} g_{i j} l_{i} l_{j}+\sum g_{i i i} l_{i}^{2} v_{i}+\sum g_{i i k} l_{i}^{2} v_{k}
$$

the contribution of $g$ to $\ln Q_{c}$ becomes

$$
\begin{array}{r}
-2(h c / k T) \Sigma\left\{g_{i i} r_{i} s_{i}^{2}+g_{i i i} r_{i}\left(1+3 r_{i}\right) s_{i}^{3}+g_{i i k} d_{k} r_{i} r_{k} s_{i}^{2} s_{k}-2 g_{i i} a_{i} r_{i}^{2} s_{i}^{3}\right\}+(h c / k T)^{2} \Sigma\left\{g _ { i i } ^ { 2 } \left(r_{i}+8 r_{i}^{2}\right.\right. \\
\left.\left.+r_{i}^{3}\right) s_{i}^{4}+2 g_{i j}^{2} r_{i} r_{j} s_{i}^{2} s_{i}^{2}+4 g_{i i} x_{i i} r_{i}^{2}\left(4+3 r_{i}\right) s_{i}^{4}+2 g_{i i} x_{i k} r_{i} r_{k}\left(1+r_{i}\right) s_{i}^{3} s_{k}\right\} .
\end{array}
$$

${ }_{7}$ For a case with 3 frequencies, for example, $x_{i i} x_{i j}$ which is not completely symmetric in the indices would include $x_{11} x_{13}$ and also $x_{33} x_{31}$. But $x_{i i} x_{i j} x_{i j}$ has complete symmetry in the indices, and $x_{33} x_{11} x_{13}$ is sufficient without $x_{33} x_{11} x_{31}$ also. 
Constants of the latter two types in eq (20) are not experimentally available at present. For a linear molecule, when there is only one doubly degenerate frequency, with $l=l_{2}$, the $-B_{v} l^{2}$ term of eq (16) is commonly absorbed into the $g_{22} l_{2}^{2}$ term. In that case, $g_{i i}$ of eq (21) represents $g_{22}+B_{v}$ of the spectroscopist.

The free energy function for a mole of a substance in the standard ideal gas state is given by

$$
-\left(F^{\circ}-E_{0}^{\circ}\right) / R T=\ln Q+(5 / 2) \ln T+(3 / 2) \ln M+\ln (2 \pi / N)^{3 / 2} k^{5 / 2} / h^{3} P_{0},
$$

$P_{0}$ being atmospheric pressure, $M$ the molecular weight, and the others known physical constants. The other functions follow by differentiation as given in appendix 5 .

A tabulation by Pennington and Kobe [6] may also be used for convenient evaluation of the more important of the contribution here treated.

\section{References}

[1] E. B. Wilson, Jr., J. Chem. Phys. 4, 525 (1936).

[2] K. F. Stripp and J. G. Kirkwood, J. Chem. Phys. 19, 1131 (1951).

[3] W. S. Benedict (with A. M. Bass), unpublished data.

[4] W. H. Stockmayer, G. M. Kavanagh, and H. S. Mickley, J. Chem. Phys. 12, 408 (1944).

[5] L. M. Milne-Thompson, The calculus of finite differences (Macmillan and Co., Ltd., London, 1933).

[6] R. E. Pennington and K. A. Kobe, J. Chem. Phys. 22, 1442 (1954).

\section{Appendices}

\subsection{Appendix 1}

The relation of the constants of eq (6) with those in

$$
\begin{aligned}
G_{v}=\sum \bar{\omega}_{i}\left(v_{i}+d_{i} / 2\right)+\sum \bar{x}_{i i}\left(v_{i}+d_{i} / 2\right)^{2}+\sum_{i<j} \bar{x}_{i j}\left(v_{i}+d_{i} / 2\right)\left(v_{j}+d_{j} / 2\right)+\sum \bar{y}_{i i i}\left(v_{i}+\bar{d}_{i} / 2\right)^{3} \\
+\sum_{i \neq j} \bar{y}_{i i j}\left(v_{i}+d_{i} / 2\right)^{2}\left(v_{j}+d_{j} / 2\right)+\sum_{i<j<k} \bar{y}_{i j k}\left(v_{i}+d_{i} / 2\right)\left(v_{j}+d_{j} / 2\right)\left(v_{k}+d_{k} / 2\right)
\end{aligned}
$$

is

$$
\begin{gathered}
\omega_{i}=\bar{\omega}_{i}+\left(d_{i}+1\right) \bar{x}_{i i}+\sum_{j} \frac{1}{2} d_{j} \bar{x}_{i j}+(1 / 4)\left(3 d_{i}^{2}+6 d_{i}+4\right) \bar{y}_{i i i} \\
+\sum_{j}\left(\frac{1}{2}\right)\left(d_{i}+1\right) d_{j} \bar{y}_{i i j}+\sum_{j}\left(d_{j}^{2} / 4\right) \bar{y}_{i j j}+\sum_{j<k}\left(d_{j} d_{k} / 4\right) \bar{y}_{i j k} \\
x_{i i}=\bar{x}_{i i}+\left(\frac{1}{2}\right)\left(3 d_{i}+6\right) \bar{y}_{i i i}+\sum_{j}\left(d_{j} / 2\right) \bar{y}_{i i j} \\
x_{i j}=\bar{x}_{i j}+\left(d_{i}+1\right) \bar{y}_{i i j}+\left(d_{j}+1\right) \bar{y}_{i j j}+\sum_{k}\left(d_{k} / 2\right) \bar{y}_{i j k} \\
y_{i i i}=\bar{y}_{i i i}, y_{i i j}=\bar{y}_{i i j} \text { and } y_{i j k}=\bar{y}_{i j k} .
\end{gathered}
$$

\subsection{Appendix 2}

To obtain eq (9) and (10), begin with the identity

$$
\sum_{v=0}^{\infty} x^{-v}=\left(1-x^{-1}\right)^{-1}
$$

Multiply by $x^{Z-1}$ and differentiate $n$ times with respect to $x$, getting

$$
\sum_{v=0}(-1)^{n} \frac{(v+n-Z) !}{(v-Z) !} x^{-v-1+Z-n}=\frac{d^{n}}{d x^{n}}\left[x^{Z}(x-1)^{-1}\right] .
$$


For the second member of the identity, let $x-1=t$, giving

$$
\frac{d^{n}}{d t^{n}}\left[(t+1)^{Z} t^{-1}\right] \quad \text { or } \quad \frac{d^{n}}{d t^{n}}\left[\sum_{r=0}^{r=Z} \frac{Z !}{(Z-r) ! r !} t^{Z-r-1}\right] .
$$

For $n>Z-1$, the only contribution is from the one term with $r=Z$, giving $\frac{d^{n}}{d t^{n}} t^{-1}$ or $(-1)^{n} n$ ! $(x-1)^{-1-n}$. Multiplying both members of the identity by $(-1)^{n} \frac{x^{1-Z+n}}{(n-Z) !}$, one has

$$
\sum_{v=0} \frac{(v+n-Z) ! x^{-v}}{(v-Z) !(n-Z) !}=\frac{n !}{(n-Z) !} x^{-Z}\left(1-x^{-1}\right)^{-1-n} .
$$

Inserting $e^{-u_{i}}$ for $x$, one may obtain eq (10) by using $n=Z+d_{i}-1$, with eq (9) as the special case given by $Z=0$.

\subsection{Appendix 3}

Functions of $v$ in terms of $(f(s)=v ! /(v-s) !$ :

$$
\begin{gathered}
v=f(1) \\
v^{2}=f(2)+f(1) \\
v^{3}=f(3)+3 f(2)+f(1) \\
v^{4}=f(4)+6 f(3)+7 f(2)+f(1) \\
v^{5}=f(5)+10 f(4)+25 f(3)+15 f(2)+f(1) \\
v^{6}=f(6)+15 f(5)+65 f(4)+90 f(3)+31 f(2)+f(1) \\
v^{3}-v^{2}=f(3)+2 f(2) \\
v^{4}-v^{3}=f(4)+5 f(3)+4 f(2) \\
v^{4}-2 v^{3}+v^{2}=f(4)+4 f(3)+2 f(2) \\
v^{5}-2 v^{4}+v^{3}=f(5)+8 f(4)+14 f(3)+4 f(2) .
\end{gathered}
$$

\subsection{Appendix 4}

Additional terms in the formulas given earlier are as follows:

Equation (12):

$$
\begin{aligned}
& -(1 / 6)(h c / k T)^{3} \sum\left\{4 x_{i i}^{3} d_{i}\left(d_{i}+1\right) r_{i}^{2}\left[1+\left(8 d_{i}+12\right) r_{i}+\left(8 d_{i}^{2}+22 d_{i}+15\right) r_{i}^{2}+\left(2 d_{i}^{2}+4 d_{i}+2\right) r_{i}^{3}\right] s_{i}^{6}\right. \\
& \quad+x_{i j}^{3} d_{i} d_{j} r_{i} r_{j}\left[1+\left(3 d_{i}+1\right) r_{i}+\left(3 d_{j}+1\right) r_{j}+d_{i}^{2} r_{i}^{2}+d_{i}^{2} r_{j}^{2}+\left(6 d_{i} d_{j}+3 d_{i}+3 d_{j}+1\right) r_{i} r_{j}+d_{i}^{2} r_{i}^{2} r_{j}\right. \\
& \left.\quad+d_{i}^{2} r_{i} r_{i}^{2}\right] s_{i}^{3} s_{j}^{3}+12 x_{i i}^{2} x_{i j} d_{i}\left(d_{i}+1\right) d_{j} r_{i}^{2} r_{j}\left[1+\left(3 d_{i}+4\right) r_{i}+\left(d_{i}+1\right) r_{i}^{2}\right] s_{i}^{5} s_{j}+6 x_{i i} x_{i j}^{2} d_{i}\left(d_{i}+1\right) d_{j} r_{i}^{2} r_{j}[2 \\
& \left.\quad+\left(2 d_{i}+1\right) r_{i}+2 d_{j} r_{j}+d_{j} r_{i} r_{j}\right] s_{i}^{4} s_{j}^{2}+3 x_{i j}^{2} x_{i k} d_{i} d_{j} d_{k} r_{i} r_{j} r_{k}\left[1+\left(2 d_{i}+1\right) r_{i}+d_{j} r_{j}+d_{j} r_{i} r_{j}\right] s_{i}^{3} s_{j}^{2} s_{k} \\
& \quad+24 x_{i i} x_{j j} x_{i j} d_{i}\left(d_{i}+1\right) d_{j}\left(d_{j}+1\right) r_{i}^{2} r_{j}^{2} s_{i}^{3} s_{j}^{3}+12 x_{i i} x_{i j} x_{i k} d_{i}\left(d_{i}+1\right) d_{j} d_{k} r_{i}^{2} r_{j} r_{k}\left(2+r_{i}\right) s_{i}^{4} s_{j} s_{k} \\
& \quad+12 x_{i i} x_{i j} x_{j k} d_{i}\left(d_{i}+1\right) d_{j} d_{k} r_{i}^{2} r_{j} r_{k} s_{i}^{3} s_{j}^{2} s_{k}+6 x_{i j} x_{i k} x_{i l} d_{i} d_{j} d_{k} d_{l} r_{i} r_{j} r_{k} r_{l}\left(1+r_{i}\right) s_{i}^{3} s_{j} s_{k} s_{l} \\
& \left.\quad+6 x_{i j} x_{i k} x_{j l} d_{i} d_{j} d_{k} d_{l} r_{i} r_{j} r_{k} r_{l} s_{i}^{2} s_{j}^{2} s_{k} s_{l}+6 x_{i j} x_{i k} x_{j k} d_{i} d_{j} d_{k} r_{i} r_{j} r_{k}\left(1+d_{i} r_{i}+d_{j} r_{j}+d_{k} r_{k}\right) s_{i}^{2} s_{j}^{2} s_{k}^{2}\right\}
\end{aligned}
$$


Equation (13):

$-(h c / k T) \sum\left\{z_{i i i i} d_{i}\left(d_{i}+1\right)\left(d_{i}+2\right)\left(d_{i}+3\right) r_{i}^{4} s_{i}^{4}+z_{i i i j} d_{i}\left(d_{i}+1\right)\left(d_{i}+2\right) d_{j} r_{i}^{3} r_{j} s_{i}^{3} s_{j}\right.$

$\left.+z_{i i j k} d_{i}\left(d_{i}+1\right) d_{j} d_{k} r_{i}^{2} r_{j} r_{k} s_{i}^{2} s_{j} s_{k}+z_{i i j j} d_{i}\left(d_{i}+1\right) d_{j}\left(d_{j}+1\right) r_{i}^{2} r_{i}^{2} s_{i}^{2} s_{j}^{2}+z_{i j k l} d_{i} d_{j} d_{k} d_{l} r_{i} r_{j} r_{k} r_{l} s_{i} s_{j} s_{k} s_{l}\right\}$

$+(1 / 2)(h c / k T)^{2} \sum\left\{12 x_{i i} y_{i i i} d_{i}\left(d_{i}+1\right)\left(d_{i}+2\right) r_{i}^{3}\left[1+\left(d_{i}+1\right) r_{i}\right] s_{i}^{5}+4 x_{i i} y_{i i j} d_{i}\left(d_{i}+1\right) d_{j} r_{i}^{2} r_{j}[1\right.$

$\left.+2\left(d_{i}+1\right) r_{i}\right] s_{i}^{4} s_{j}+4 x_{i i} y_{j j i} d_{i}\left(d_{i}+1\right) d_{j}\left(d_{j}+1\right) r_{i}^{2} r_{j}^{2} s_{i}^{3} s_{j}^{2}+4 x_{i i} y_{i j k} d_{i}\left(d_{i}+1\right) d_{j} d_{k} r_{i}^{2} r_{j} r_{k} s_{i}^{3} s_{j} s_{k}$

$+6 x_{i j} y_{i i i} d_{i}\left(d_{i}+1\right)\left(d_{i}+2\right) d_{j} r_{i}^{3} r_{j} s_{i}^{4} s_{j}+2 x_{i j} y_{i i j} d_{i}\left(d_{i}+1\right) d_{j} r_{i}^{2} r_{j}\left[2+d_{i} r_{i}+2 d_{j} r_{j}\right] s_{i}^{3} s_{j}^{2}$

$+4 x_{i j} y_{i i k} d_{i}\left(d_{i}+1\right) d_{j} d_{k} r_{i}^{2} r_{j} r_{k} s_{i}^{3} s_{j} s_{k}+2 x_{i j} y_{k k i} d_{i} d_{j} d_{k}\left(d_{k}+1\right) r_{i} r_{j} r_{k}^{2} s_{i}^{2} s_{j} s_{k}^{2}+2 x_{i j} y_{i k l} d_{i} d_{j} d_{k} d_{l} r_{i} r_{j} r_{k} r_{l} s_{i}^{2}$

$\left.s_{j} s_{k} s_{l}+2 x_{i j} y_{i j k} d_{i} d_{j} d_{k} r_{i} r_{j} r_{k}\left(1+d_{j} r_{i}+d_{j} r_{j}\right) s_{i}^{2} s_{j}^{2} s_{k}\right\}$

Equation (14):

$\Sigma\left\{a_{i} a_{i i} d_{i} r_{i}\left[1+\left(1+2 d_{i}\right) r_{i}\right] s_{i}^{3}+a_{i} a_{i j} d_{i} d_{j} r_{i} r_{j} s_{i}^{2} s_{j}+(1 / 6) a_{i}^{3} d_{i} r_{i}\left(1+r_{i}\right) s_{i}^{3}+a_{i i i} d_{i} r_{i}\left[1+\left(1+3 d_{i}\right) r_{i}\right.\right.$

$\left.\left.+d_{i}^{2} r_{i}^{2}\right] s_{i}^{3}+a_{i i j} d_{i} d_{j} r_{i} r_{j}\left(1+d_{i} r_{i}\right) s_{i}^{2} s_{j}+a_{i j k} d_{i} d_{j} d_{k} r_{i} r_{j} r_{k} s_{i} s_{j} s_{k}\right\}$

Equation (15):

$-(h c / k T) \Sigma\left\{a_{i i} x_{i i} d_{i}\left(d_{i}+1\right) r_{i}^{2}\left[4+\left(4 d_{i}+2\right) r_{i}\right] s_{i}^{4}+2 a_{i j} x_{i i} d_{i}\left(d_{i}+1\right) r_{i}^{2} r_{j} s_{i}^{3} s_{j}+a_{i i} x_{i j} d_{i} d_{j} r_{i} r_{j}[1\right.$

$\left.\left.+\left(2 d_{i}+1\right) r_{i}\right] s_{i}^{3} s_{j}+a_{i j} x_{i j} d_{i} d_{j} r_{i} r_{j}\left[1+d_{i} r_{i}+d_{j} r_{j}\right] s_{i}^{2} s_{i}^{2}+a_{i j} x_{k j} d_{i} d_{j} d_{k} r_{i} r_{j} r_{k} s_{i} s_{j}^{2} s_{k}\right\}$

$+\frac{1}{2}(h c / k T)^{2} \Sigma\left\{4 a_{i} x_{i i}^{2} d_{i}\left(d_{i}+1\right) r_{i}^{2}\left[1+\left(3 d_{i}+4\right) r_{i}+\left(d_{i}+1\right) r_{i}^{2}+\left(d_{i}^{2}+d_{i}\right) r_{i}^{3}\right] s_{i}^{5}+a_{i} x_{i j}^{2} d_{i} d_{j} r_{i} r_{j}[1\right.$

$\left.+\left(2 d_{i}+1\right) r_{i}+d_{j} r_{j}+d_{j} r_{i} r_{j}\right] s_{i}^{3} s_{j}^{2}+4 a_{i} x_{i i} x_{i j} d_{i}\left(d_{i}+1\right) d_{j} r_{i}^{2} r_{j}\left(2+r_{i}\right) s_{i}^{4} s_{i}+4 a_{j} x_{i i} x_{i j} d_{i}\left(d_{i}\right.$

$\left.+1) d_{j} r_{i}^{2} r_{j} s_{i}^{3} s_{j}^{2}+2 a_{i} x_{i j} x_{i k} d_{i} d_{j} d_{k} r_{i} r_{j} r_{k}\left(1+r_{i}\right) s_{i}{ }^{3} s_{j} s_{k}+2 a_{j} x_{i j} x_{i k} d_{i} d_{j} d_{k} r_{i} r_{j} r_{k} s_{i} s_{j}^{2} s_{k}\right\}$

Equation (21):

$-(1 / 3)(h c / k T)^{3} \sum\left\{g_{i i}^{3}\left(r_{i}+50 r_{i}^{2}+194 r_{i}^{3}+50 r_{i}^{4}+r_{i}^{5}\right) s_{i}^{6}+6 x_{i i} g_{i i}^{2} r_{i}^{2}\left(16+99 r_{i}+70 r_{i}^{2}+3 r_{i}^{3}\right) s_{i}^{6}\right.$

$+3 x_{i j} g_{i i}^{2} d_{j} r_{i} r_{j}\left(1+19 r_{i}+19 r_{i}^{2}+r_{i}^{3}\right) s_{i}^{5} s_{j}+12 x_{i j} g_{i i} g_{j j} r_{i} r_{j}\left(1+r_{i}\right)\left(1+r_{j}\right) s_{i}^{3} s_{j}^{3}$

$+12 x_{i i}^{2} g_{i i} r_{i}^{2}\left(4+55 r_{i}+70 r_{i}^{2}+9 r_{i}^{3}\right) s_{i}^{6}+12 x_{i i} x_{i j} g_{i i} d_{j} r_{i}^{2} r_{j}\left(8+17 r_{i}+3 r_{i}^{2}\right) s_{i}^{5} s_{j}$

$+3 x_{i j}^{2} g_{i i} d_{j} r_{i} r_{j}\left[1+8 r_{i}+5 r_{i}^{2}+d_{j}\left(r_{j}+4 r_{i} r_{j}+r_{i}^{2} r_{j}\right)\right] s_{i}^{4} s_{j}^{2}+12 x_{i j} x_{j j} g_{i i} d_{j}\left(d_{j}+1\right) r_{i} r_{j}^{2}\left(1+r_{i}\right) s_{i}^{3} s_{j}^{3}$

$\left.+6 x_{i j} x_{i k} g_{i \imath} d_{j} d_{k} r_{i} r_{j} r_{k}\left(1+4 r_{i}+r_{i}^{2}\right) s_{i}^{4} s_{j} s_{k}+6 x_{i j} x_{j k} g_{i i} d_{j} d_{k} r_{i} r_{j} r_{k}\left(1+r_{i}\right) \varepsilon_{i}^{3} s_{j}^{2} s_{k}\right\}$

\subsection{Appendix 5}

For a term of $\ln Q$ of the form

$$
z=x u^{p} f_{1} \prod_{\imath} r_{i}^{m_{i}} s_{i}^{n_{i}}=W f_{1},
$$

with $f_{1}=1+\sum_{i} t_{i} r_{i}^{q_{i}}$ and $u=h c / k T$ and with $x, p, m_{i}, n_{i}, t_{i}$, and $q_{i}$ constants, the contributions to the various thermodynamic functions are: For $-\left(F^{\circ}-E_{0}^{\circ}\right) / R T, z=W f_{1}$; for $\left(H^{\circ}-E_{0}^{\circ}\right) / R T$, $-u d z / d u=W h_{2}$; for $C_{p}^{\circ} / R, u^{2} d^{2} z / d u^{2}=W h_{3}$; with $h_{2}=f_{1} g_{1}+f_{2}, h_{3}=f_{1} g_{2}+2 f_{2} g_{1}+f_{3}$, where

$$
\begin{aligned}
& f_{2}=\sum_{i} t_{i} q_{i} u_{i} r_{i}^{q_{i}} \\
& f_{3}=\sum_{i} t_{i} q_{i}^{2} u_{i}^{2} r_{i}^{q_{i}} \\
& g_{1}=-p+\sum_{i} n_{i} u_{i} r_{i} s_{i}+\sum m_{i} u_{i} \\
& g_{2}=-p+\sum_{i} n_{i} u_{i}^{2} r_{i} s_{i}^{2}+g_{1}^{2} .
\end{aligned}
$$

Washington, January 11, 1956. 